\title{
Infratemporal Fossa, Masticator Space and Parapharyngeal Space: Can the Radiologist and Surgeon speak the Same Language?
}

\author{
Supreeta Arya, Pawan Rane, Anil D’Cruz
}

\begin{abstract}
Imaging is an integral part of head and neck cancer staging and assessing resectability. Accurate perception of imaging information by the clinician is possible only by effective communication between radiologist and surgeon. Traditionally, the radiologist studies the head neck region with two dimensional cross-sectional imaging. The surgeon perceives the head and neck region by real-time experience at surgery as a threedimensional (3D) space. Advances in computed tomography (multidetector CT) provide multiplanar and 3D reformations as added tools to facilitate understanding the complex anatomy and pathology and improve accuracy in staging. Despite these aids, accurate information requires a precise understanding of the different nomenclature of suprahyoid spaces used by the radiologist and clinician. While clinicians are familiar with infratemporal fossa (ITF), radiologists are familiar with masticator space (MS). Although these have been used interchangeably in the staging systems, the subtle difference needs to be understood. Literature also has differing definitions of the parapharyngeal space (PPS). This article describes these spaces, the varied definitions of PPS and the implications of involvement of structures of the ITF and MS on T staging of head neck cancers. A practical approach to the differential diagnoses of parapharyngeal lesions is also described.
\end{abstract}

Keywords: Infratemporal fossa, Masticator space, Parapharyngeal space.

How to cite this article: Arya S, Rane P, D'Cruz A. Infratemporal Fossa, Masticator Space and Parapharyngeal Space: Can the Radiologist and Surgeon speak the Same Language? Int J Otorhinolaryngol Clin 2012;4(3):125-135.

Source of support: Nil

Conflict of interest: None declared

\section{BACKGROUND}

The TNM staging of head and neck cancers is based on clinical examination as well as radiological staging. While labeling extent of disease in the suprahyoid neck, the radiologist and the surgeon often vary in their nomenclature of the various spaces of the suprahyoid neck. This could inadvertently result in imprecise communication. This difference results due to the fact that the radiologist encounters the spaces virtually and traditionally views these regions in serial axial sections with cross-sectional imaging. However, the surgeon has a three-dimensional (3D) understanding of this region based on the real-time experience at surgery. The clinician requires to translate the information obtained with cross-sectional imaging into a 3D image. Advances in computed tomographic (CT) imaging with the advent of multidetector scanners (MDCT) scanners have enabled volume acquisition of data and reconstruction with thin slices. The resultant high quality coronal, sagittal and oblique reformations, as well as volume rendered and 3D reformations are additional aids in the understanding of the complex anatomy and pathology of the head and neck region. Yet uniformity of nomenclature or clear understanding of the different terminology can further improve communication.

The conventional radiologic classification of the suprahyoid neck region is based on the layers of cervical fascia cleaving this region into several spaces (Fig. 1). Such classification is useful to the diagnostic radiologist because (i) it helps decide the epicenter of disease origin and (ii) it helps arrive at a list of differential diagnoses peculiar to each space. The clinician is often not familiar with this classification. The differing terminology used by the radiologist and the surgeon needs to be described to improve understanding of disease spread. This article describes the anatomy of the infratemporal fossa (ITF) and masticator space (MS), and the various definitions of the parapharyngeal space (PPS) to improve communication between the radiologist and the surgeon. The article also demonstrates the implications of involvement of the structures of ITF and MS on the T staging (AJCC 7th edition) in various head and neck cancers. Lastly the differential diagnoses of PPS masses is discussed.

\section{Masticator Space}

The MS is a term more familiar to the radiologist. Juvara first described this space while the first reference to MS was made by Coller and Yglesias. ${ }^{1}$

\section{Anatomy}

The MS as the name implies consists of the masticator muscles (medial and lateral pterygoid, temporalis and masseter) and the posterior body and ramus of the mandible along with the mandibular nerve and the internal maxillary artery. This space is located anterolateral to the PPS (Fig. 1). Unlike the ITF it is clearly a fascia enclosed space. The investing layer of cervical fascia splits at the lower border 


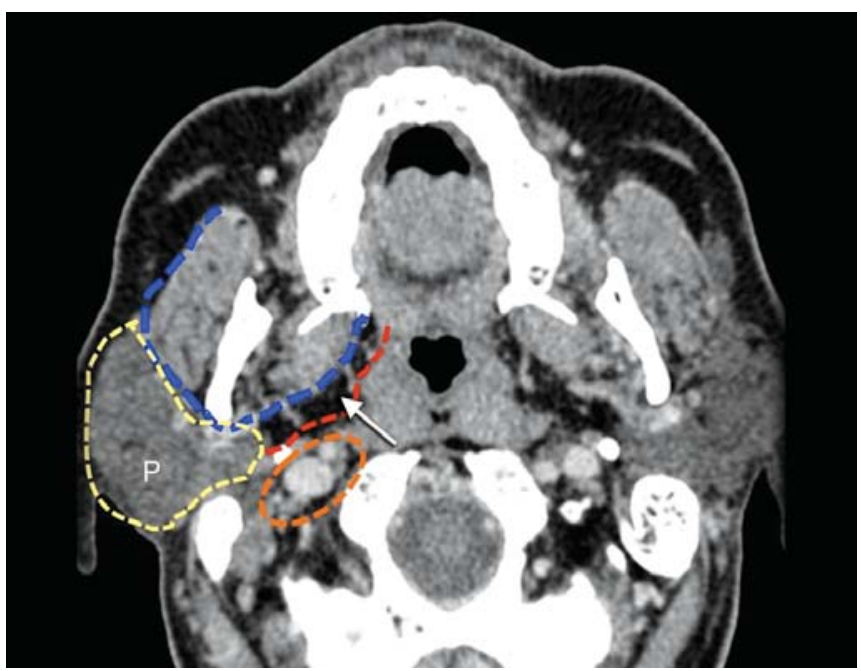

Fig. 1: Axial CT section shows outlines of various spaces (Harnsberger classification), MS (blue), parotid space (yellow), carotid space (orange) and the fat containing PPS between red and blue lines (arrow)

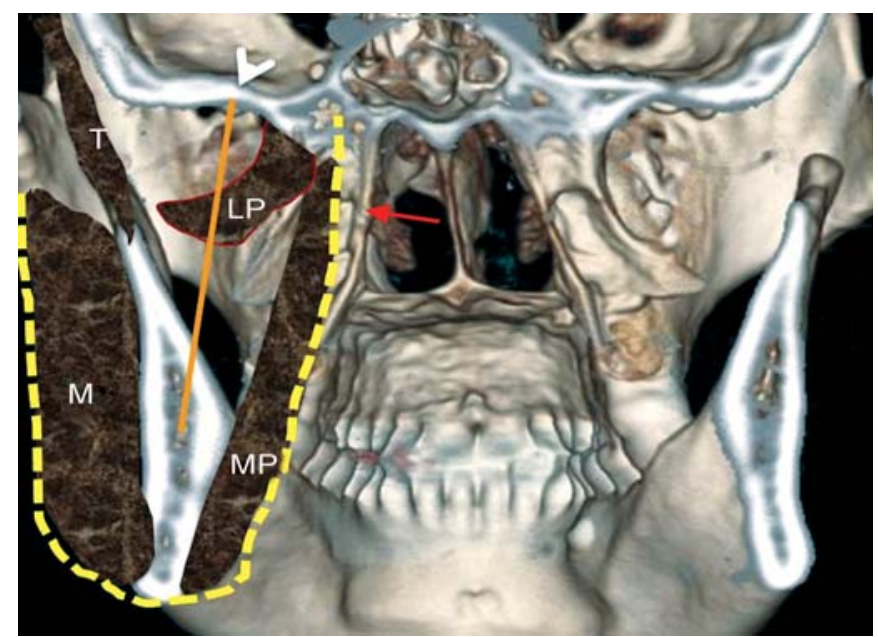

Fig. 2: Infrazygomatic MS-Outlines (yellow) and contents: medial pterygoid (MP), masseter (M) and temporalis ( $T$ ) and lateral pterygoid (LP) muscles. Also shown are medial pterygoid plate (red arrow), foramen ovale (white arrowhead) and mandibular nerve (orange line)

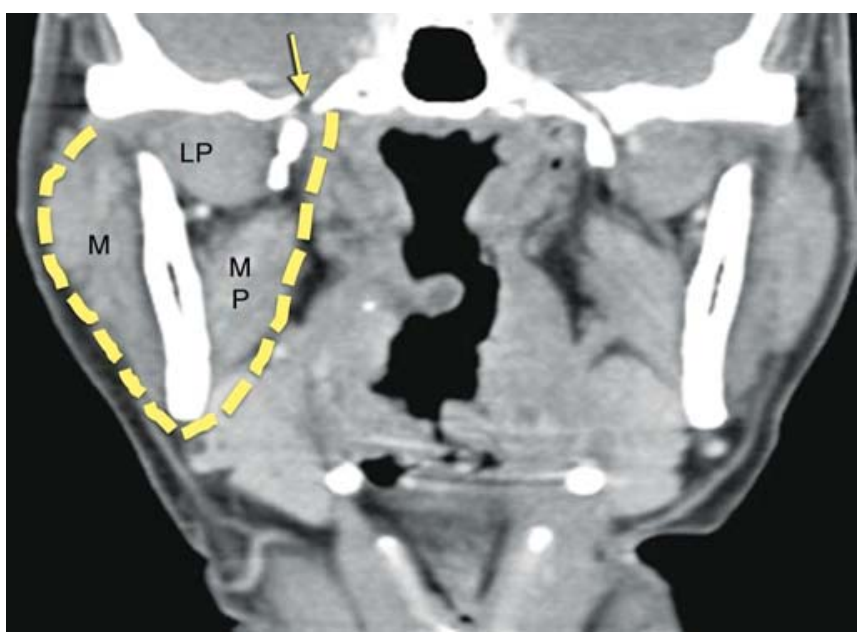

Fig. 3: MS boundaries (yellow dotted lines) on a coronal CT section (soft tissue window) with contents, lateral pterygoid (LP), medial pterygoid (MP), masseter (M). Arrow shows foramen ovale of the mandible into two layers that enclose the MS. The superficial layer lies superficial to the masseter and inferior tendon of temporalis and merges with the fascia covering the temporalis in the scalp. The deep or medial layer passing deep to the medial pterygoid muscle attaches to the skull base from the root of the medial pterygoid plate to the sphenoid spine just medial to the foramen ovale. The resultant MS is a pyramidal-shaped space with its base toward the skull base and the apex at the lower border of the mandible (Fig. 2). The foramina ovale (Figs 2 and 3) and spinosum lie in the roof of this space. The inferior alveolar nerve that lies within the canal of the mandible exits at the mandibular foramen and continues cranially as the mandibular nerve within the MS to reach the foramen ovale. $^{2-4}$

The anterior boundary of the MS is somewhat incomplete where it borders the retroantral buccal fat. While

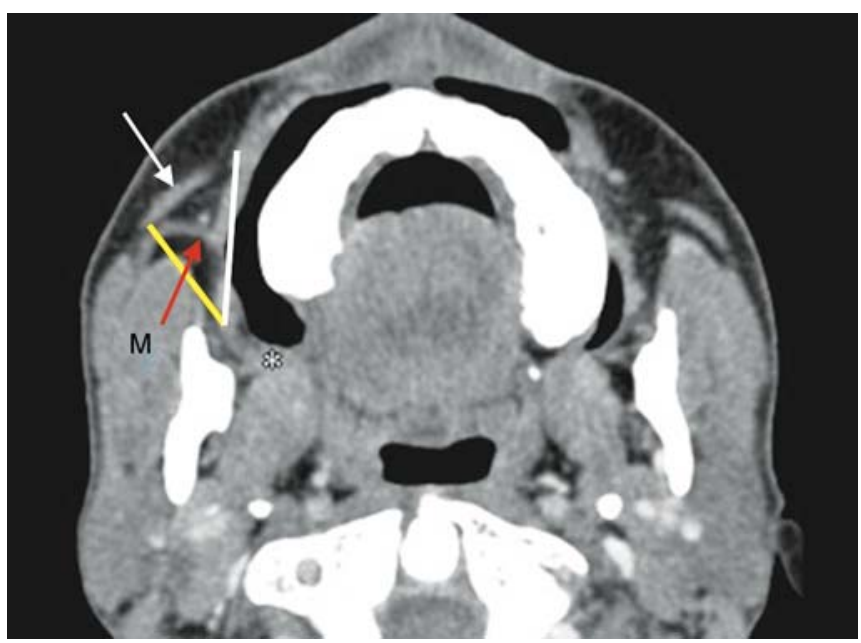

Fig. 4: Buccal space (BS). Anterior margin is zygomaticus major (arrow); medial boundary is buccinator (white line); posterior margin (yellow line) is along masseter (M). The enclosed BS contains terminal parotid duct (red arrow)

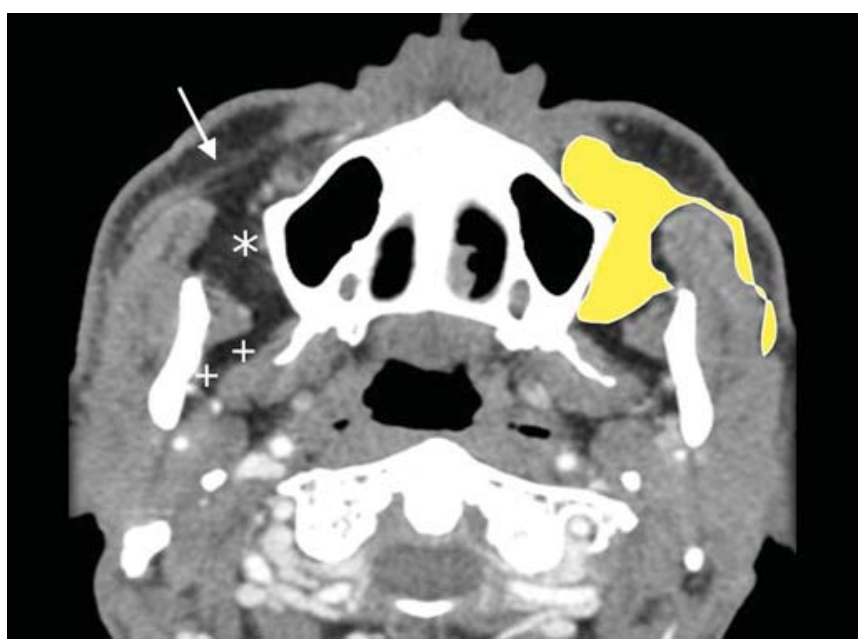

Fig. 5: Upper buccal space (yellow outlined space). Arrow shows zygomaticus major. $\left({ }^{*}\right)$ shows retroantral buccal fat, $(++)$ shows fat in anterior MS 
some include the latter as a part of the MS, others define it as a part of a separate buccal space. The buccal space is located lateral to the buccinator, medial to the zygomaticus major and anterior to the masseter. ${ }^{5}$ It contains the terminal part of parotid duct. Its posterior extension is the retroantral fat pad that reaches up to the anterior margin of the MS (Figs 4 and 5).

The bulk of the MS is formed by the medial pterygoid muscle which originates from the medial surface of the lateral pterygoid plate and passes downward and laterally to insert at the medial surface of the vertical mandibular ramus at the mandibular angle (Fig. 2). The fascia of this muscle contributes to the medial fascial boundary of the MS. The lateral pterygoid muscle is lesser in bulk and lies freely within the MS (Figs 2 and 3). It has two heads of origin, the upper head from the infratemporal surface and infratemporal crest of the greater wing of sphenoid. The lower head arises from the lateral surface of the lateral pterygoid plate. It then passes backward and laterally in an almost horizontal plane to insert into the condyle of the

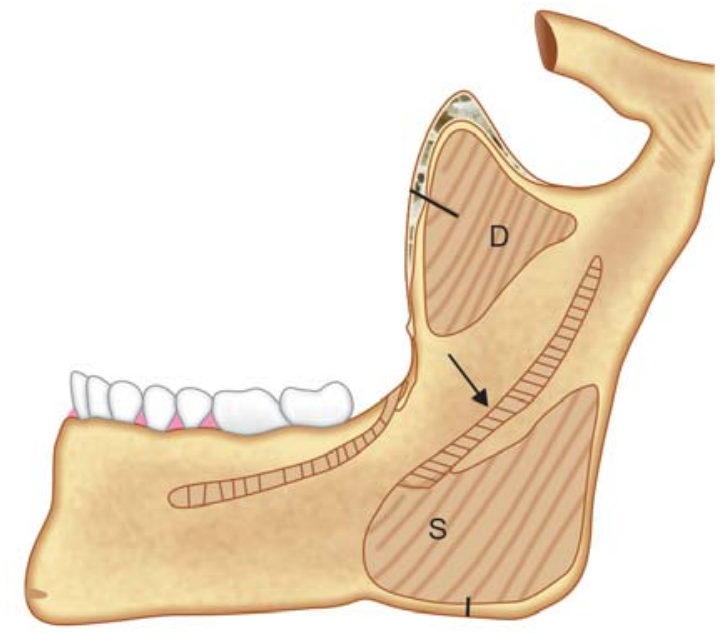

Fig. 6: Sagittal line diagram shows parts of the masseter; the superficial part (S), deep part (D) and middle part (arrow)
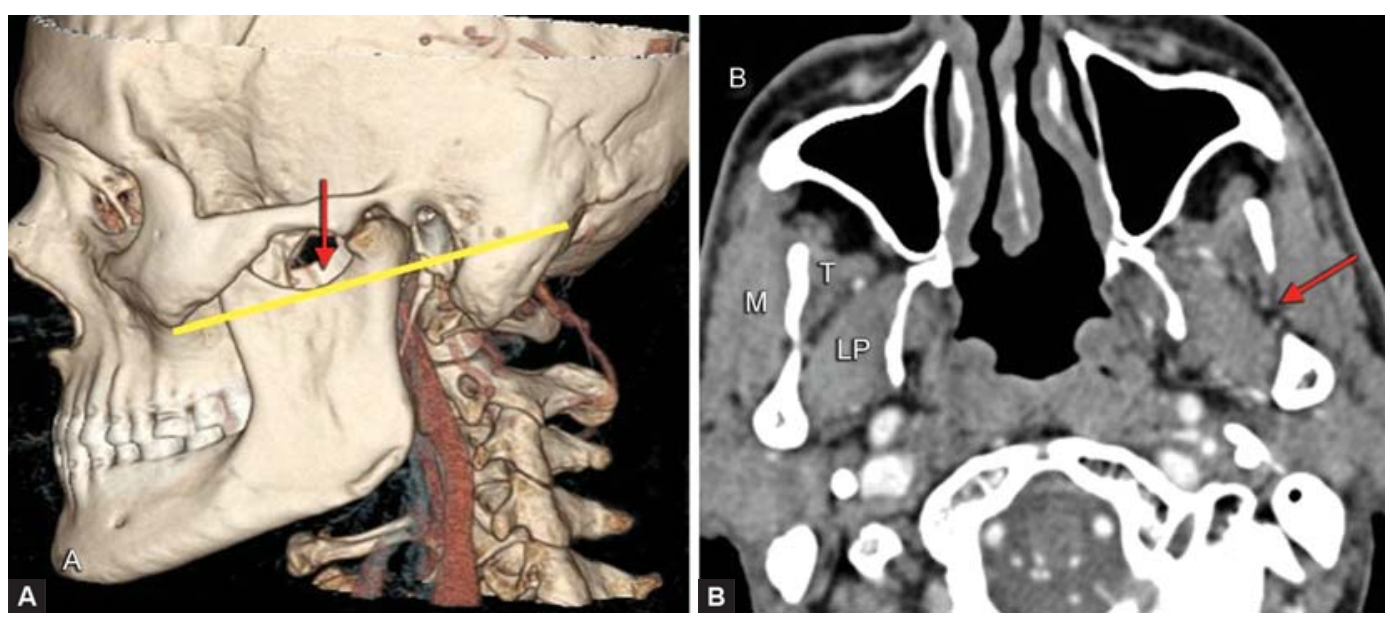

Figs 7A and B: (A) Volume rendered 3D CT image and (B) axial CT section show mandibular notch (arrow) with plane dividing supranotch and infranotch MS (line). B shows section above notch with temporalis (T), lateral pterygoid (LP) and masseter (M) mandible (a depression on its medial surface called the pterygoid fovea). The temporalis has a fan-shaped origin from the temporal fossa and from the deep part of temporal fascia. It converges into a narrow tendinous insertion at the medial surface of the coronoid process of the mandible (Fig. 2). The masseter has three layers, the superficial being the largest, that arises from the maxillary process of the zygomatic bone and the anterior two-thirds of the zygomatic arch. The middle and central parts arise from the zygomatic arch (Figs 2 and 6). The superficial part inserts into the lower posterior part of outer surface of the vertical ramus near the mandibular angle. The middle part inserts into the central part of outer surface of vertical ramus. The deep part inserts into the outer surface of upper part of mandibular ramus and the coronoid process. ${ }^{1-4}$

Radiological literature often subdivides the MS using the zygomatic arch as the horizontal division plane into (i) suprazygomatic MS which is synonymous with the temporal fossa while, (ii) infrazygomatic MS is referred simply as the MS. On the other hand surgical literature sometimes subdivides the MS into subspaces: ${ }^{3}$ (i) The masseteric space between the masseter muscle and ramus of mandible, (ii) the pterygoid space between the pterygoid muscles and ramus, (iii) the superficial temporal space between the superficial temporal fascia and temporalis muscle, and the deep temporal space between the deep temporal fascia and temporal bone. Correlating the radiological and surgical classifications, the masseteric and pterygoid spaces are in the MS (infrazygomatic region) while the superficial and deep temporal spaces are parts of the suprazygomatic MS or temporal fossa.

Another division of the MS has relevance to evaluating posterior spread of disease in oral squamous cancers. A transaxial plane passing through the notch between coronoid and condyloid process is a key landmark to classify disease 
above and below as supranotch and infranotch disease (Figs 7A and B). ${ }^{6}$ Thus, the lateral pterygoid muscle and the tendon of temporalis lie in the supranotch compartment, while the bulk of the masseter and medial pterygoid muscle lie in the infranotch MS. While the upper two-thirds of the pterygoid plates lie in the supranotch compartment, the lower parts lie in the infranotch MS.

\section{Teaching Point 1}

MS is a fascial lined space containing all four masticator muscles, the posterior body and ramus of mandible and the mandibular nerve.

\section{Parapharyngeal Space}

\section{Anatomy}

Cummings refers to the PPS also as the lateral pharyngeal or pharyngomaxillary space. The PPS is an inverted pyramid with its base against the skull base and its apex inferiorly at the junction of the posterior belly of the digastric muscle and greater cornu of the hyoid bone (Fig. 8). Anteriorly the pterygomandibular raphe and the medial pterygoid muscle form the boundaries while the prevertebral fascia bounds it posteriorly. The medial boundary is formed by the superior constrictor, tensor and levator veli palatini muscles. The parotid gland, mandible, and lateral pterygoid muscle form the lateral margins. ${ }^{3}$

In radiological literature, there are two definitions of the PPS and the radiologist and surgeon at each institute need to understand each other's nomenclature. Harnsberger classifies the suprahyoid neck spaces based on the cleaving of this region by the layers of deep cervical fascia. ${ }^{2}$ There are three layers of deep cervical fascia, namely the investing layer, the middle layer and the deep layer (also called

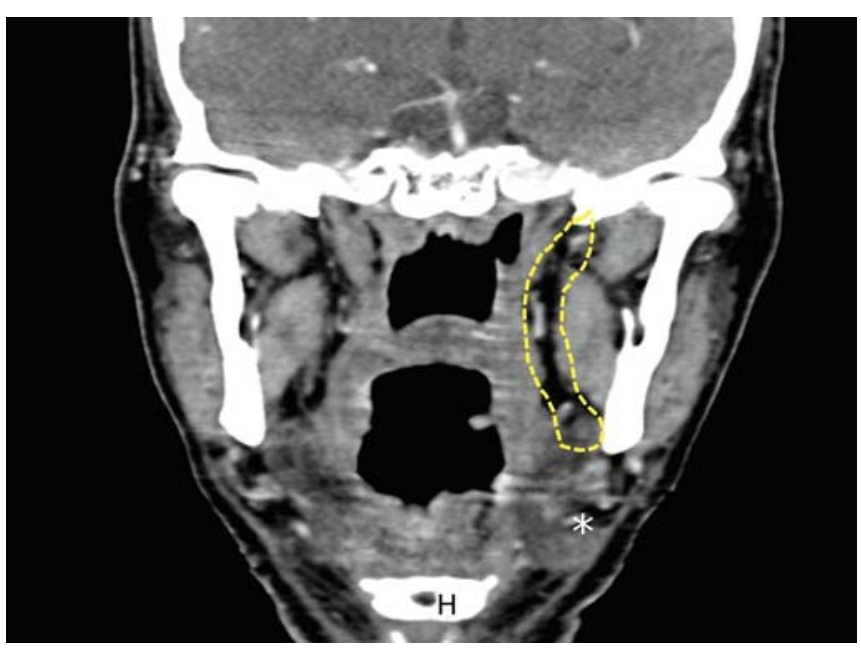

Fig. 8: The PPS extending from base skull to submandibular space $\left(^{*}\right)$ reaching up to greater cornu of hyoid bone $(\mathrm{H})$ prevertebral fascia). In this classification, the fat space lying lateral to the pharynx and the middle layer of cervical fascia (also called buccopharyngeal fascia) is referred to as the PPS (Fig. 1). This space is located posteromedial to the MS. In this definition, the carotid sheath and the neural structures within it, lying posterior to the styloid process, belong to the 'carotid space' (see Fig. 1). The deep lobe of the parotid is not a content of the PPS in this definition and belongs to the parotid space (see Fig. 1). The contents of the PPS in this definition are fat, salivary gland rests in the fat, pterygoid venous plexus, and ascending pharyngeal artery. This classification system has a unique advantage to the radiologist to arrive at and narrow the list of differential diagnoses. $^{2}$

However, Som and Curtin have an alternate definition of the PPS, more familiar to the clinicians. ${ }^{1}$ In this definition, the tensor-vascular styloid fascia divides the space posteromedial to the MS into two compartments: an anterior prestyloid compartment and a posteriorly located poststyloid compartment (Figs 9 and 10A). The contents of the prestyloid compartment are fat, deep lobe of the parotid, ascending pharyngeal artery and pterygoid venous plexus (Table 1 and Fig. 10B). In this radiological definition of the prestyloid PPS, nodes and muscle are not a content although Cummings mentions styloglossus as a component of the prestyloid PPS. The former is justified because the list of the differential diagnoses of masses in the prestyloid PPS (discussed later) does not include de novo masses of muscular origin or adenopathy. The poststyloid compartment contains neurovascular structures: Carotid artery, internal jugular vein, sympathetic chain, and cranial nerves IX, X, XI, and XII along with paraganglionic tissue (Table 1). ${ }^{1-3}$ The poststyloid or retrostyloid PPS corresponds to the 'carotid space' in the Harnsberger definition.

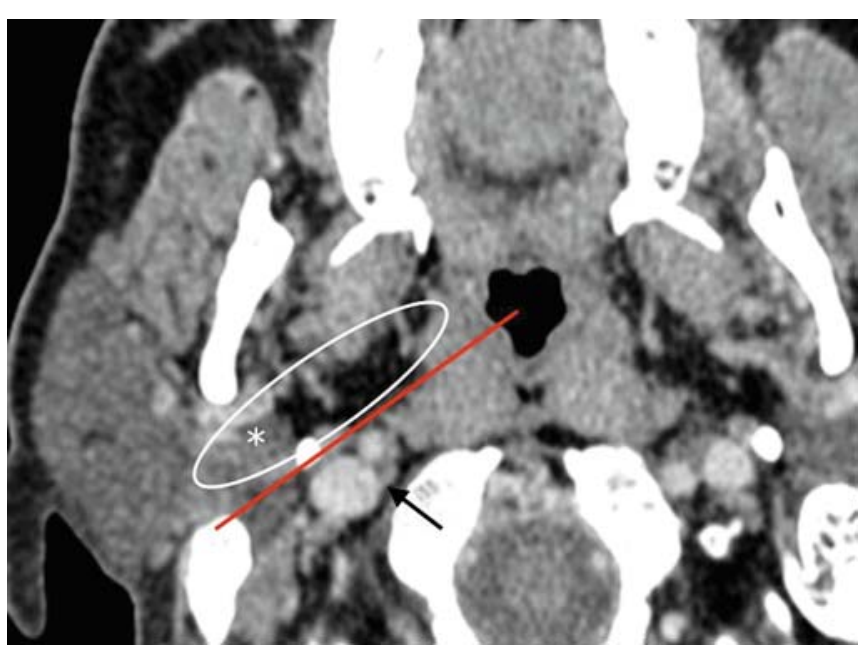

Fig. 9: Axial CT section showing divisions of PPS by line passing through styloid process into prestyloid space (encircled) containing deep lobe of parotid $\left({ }^{*}\right)$ and parapharyngeal fat and poststyloid space containing neurovascular structures (arrow) 


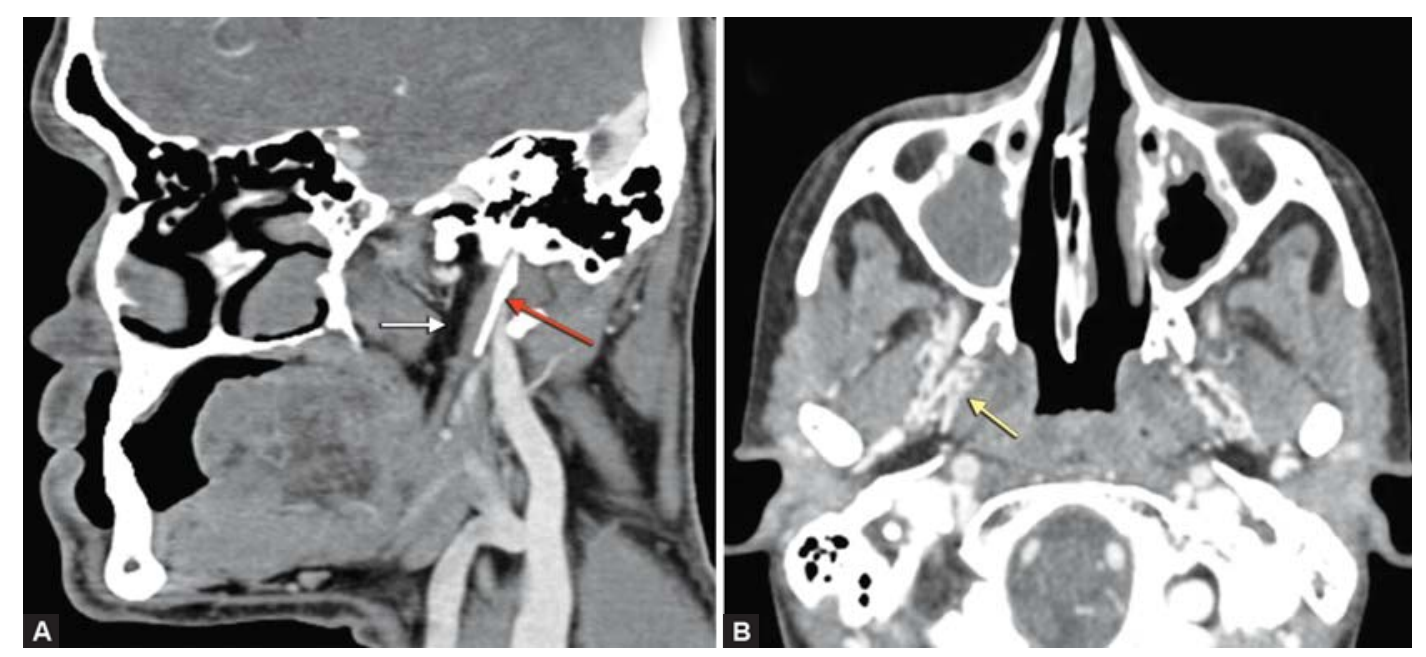

Figs 10A and B: (A) Sagittal reformat showing fat containing prestyloid PPS (white arrow) and styloid process (red arrow).

(B) Arrow shows a prominent pterygoid venous plexus, a content of prestyloid PPS

\section{Infratemporal Fossa}

The exact boundaries of the ITF are a subject of controversy. All definitions agree that is an irregular nonfascial lined space lying medial to the inner surface of the vertical ramus of the mandible and the zygomatic arch (Figs 11 and 12).
The boundaries defined by Cummings are as follows; anteriorly, by the posterior surface of the maxilla and the inferior orbital fissure; posteriorly, by the mastoid and tympanic portions of the temporal bone; and superiorly by the inferior surfaces of the greater wing of the sphenoid
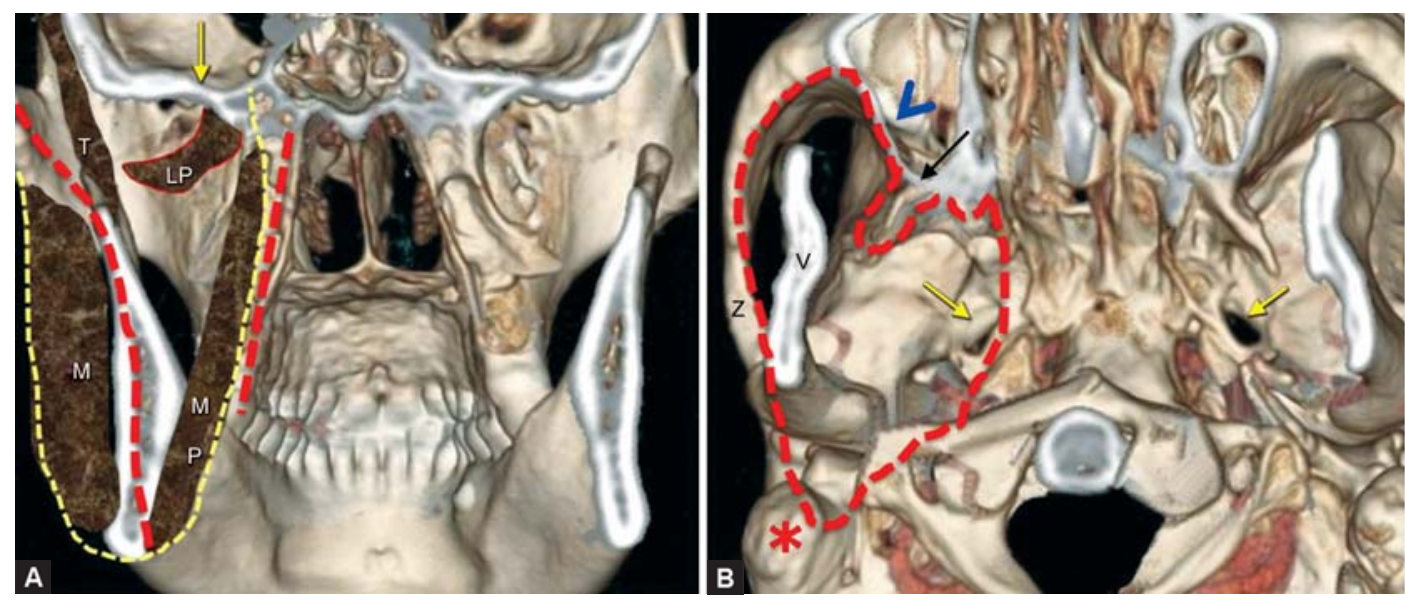

Figs 11A and B: ITF(A) 3D CT coronal view shows ITF (red lines) containing medial pterygoid (MP), lateral pterygoid (LP) muscles with foramen ovale (arrow) in roof. Compare with (infrazygomatic) masticator space (yellow) with contents MP, LP and masseter. (B) Axial view . ITF is medial to zygomatic arch (Z) and vertical ramus (V) reaching from posterior wall of maxilla (arrowhead) to mastoid process $\left({ }^{*}\right)$. Medial limit is lateral pterygoid plate (black arrow) and pharyngeal wall. Arrows show foramen ovale

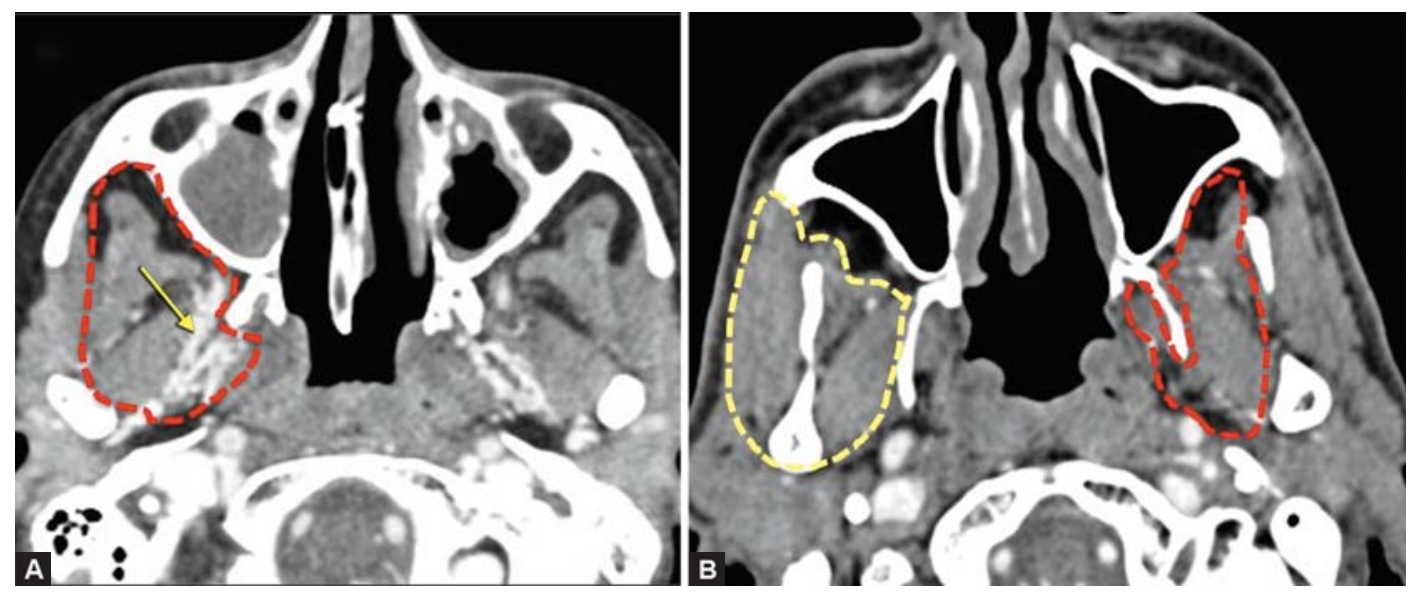

Figs 12A and B: Boundaries of ITF (red) and MS (yellow) (A) at zygomatic arch level and (B) at mandibular notch level. Note the pterygoid venous plexus (arrow) in prestyloid PPS (part of ITF) 


\begin{tabular}{ll}
\hline \multicolumn{1}{c}{ Table 1: Contents of PPS } \\
\hline Prestyloid PPS & Poststyloid PPS \\
\hline Fat & ICA \\
Salivary gland rests & IJV \\
Deep lobe of parotid & Paraganglionic tissue (located \\
& posterior to the vessels) \\
Pterygoid venous plexus & IX, X, XI, XII cranial nerves \\
& (all are located posterior to \\
& vessels, except for IX nerve \\
& that is anterior to the vessels) \\
& Sympathetic plexus (located \\
along the medial fascial wall of \\
Ascending pharyngeal artery \\
\end{tabular}

and the squamous portion of the temporal bone. The ITF communicates with the temporal fossa cranially. Laterally the ITF is bounded by the zygomatic arch and ascending ramus of the mandible. ${ }^{3}$

The medial boundary varies in different definitions. Gray’s anatomy describes the medial boundary as the lateral pterygoid plate, the pharynx, the tensor and levator veli palatini. Cummings describes the medial boundary as the sphenoid pterygoid process, lateral portion of the clivus, first cervical vertebra and inferior surface of the petrous portion of the temporal bone. ${ }^{3,4}$ The fossa is regarded as lacking an anatomical floor in some definitions with the lateral lower limit at the insertion of the medial pterygoid to the mandible. Cummings describes the inferior boundary as the superior limit of the posterior belly of the digastric muscle.

The ITF communicates through the inferior orbital fissure with the orbit, through the foraminae ovale and spinosum with the middle cranial fossa, and through the pterygomaxillary fissure with the pterygopalatine fossa. The contents of the ITF are the medial and lateral pterygoid muscles, the mandibular division of the trigeminal nerve $\left(\mathrm{V}_{3}\right)$, chorda tympani branch of facial nerve, otic ganglion, internal maxillary artery and pterygoid venous plexus. ${ }^{3,4}$ The masseter is not considered a content of the ITF in most definitions.

\section{Relation between ITF, PPS and MS}

A clear perception of the spaces described by the terms ITF, MS and PPS is needed for optimal understanding of disease spread. Although in the current lexicon of AJCC T staging for head neck cancers, ITF and MS are used interchangeably, the anatomical definitions are clearly different. Strictly speaking, the nonfascial lined ITF encompasses the medial MS (MS excluding the masseter), a part of the prestyloid compartment of PPS (the pterygoid plexus containing parapharyngeal fat) reaching up to the lateral pharyngeal wall and the retroantral buccal fat (buccal space) ${ }^{4}$ (Figs 5, 12 and 13). The foraminae ovale and spinosum are in the

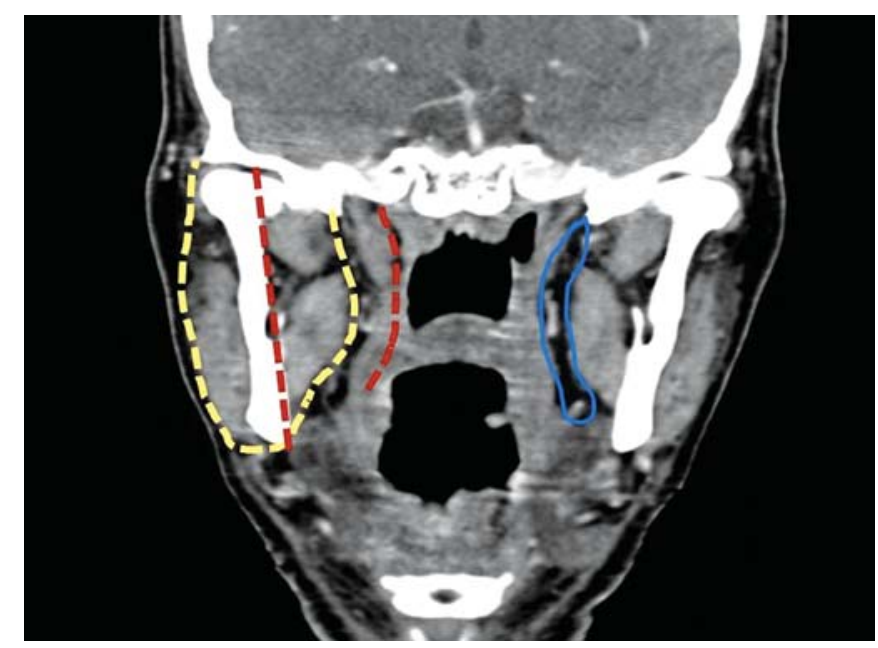

Fig. 13: Coronal CT section shows relation between ITF (red), MS (yellow) and PPS (blue). ITF has no anatomical floor

roof of the part of the MS located within the ITF (Figs 2, 3 and $11 \mathrm{~A}$ and B). The PPS is a space medial and posterior to the MS separated by the deep layer of investing cervical fascia (Figs 1 and 13) and the two spaces do not overlap. The poststyloid PPS lies posterior to both MS and ITF (Figs 1 and 9). The medial prestyloid PPS is within the ITF (Figs 12 and 13).

\section{Teaching Point 2}

The ITF is a nonfascial lined space containing much of the MS (except masseter), the retroantral buccal fat and the medial part of prestyloid PPS.

Infective processes, particularly abscesses tend to be initially confined to fascial lined spaces, while neoplasia is more likely to breach fascial boundaries and extend beyond compartments. Hence, the incidence of MS infections, usually of odontogenic origin (infected teeth or following dental manipulation) are much more common while ITF abscesses are uncommon. ${ }^{7}$ The venous drainage of the ITF is by the pterygoid venous plexus, which also anastomoses with the cavernous sinus, ophthalmic veins and pharyngeal venous plexus. Therefore, infection when occurring in the ITF can spread by hematogenous extension to involve the contents of the orbit and cavernous sinus. Bone erosion is more common with neoplasia than with infections. ${ }^{7}$ PPS abscesses do not arise de novo, but are due to spread from adjacent spaces like odontogenic infection from the MS, or spread of tonsillitis or parotitis. ${ }^{1}$

Malignancy more commonly spreads across fascial boundaries and involvement of the components of a larger compartment like the ITF is more common. Gingival, buccal and retromolar trigone cancers can spread across the buccinator to the buccal space and then posteriorly into the MS as well as pterygoid plates (T4b disease). Invasion of the masseter and medial pterygoid by oral cancers is 
Table 2: AJCC 7th edition T staging variations with respect to ITF and MS structures

\begin{tabular}{lll}
\hline Region & T stage & T stage \\
\hline $\begin{array}{l}\text { Oral cavity } \\
\text { Oropharynx }\end{array}$ & MS or pterygoid plates or skull base-T4b & Mandible erosion* (body or ramus)—T4a \\
& $\begin{array}{l}\text { Lateral pterygoid muscle, pterygoid plates, } \\
\text { lateral nasopharynx or skull base invasion_T4b }\end{array}$ & Medial pterygoid muscle invasion-T4a \\
Nasopharynx & MS or ITF invasion-T4 & PPS invasion—T2b \\
Maxillary sinus & Nasopharynx or clivus-T4b & Pterygoid plates or ITF-T4a \\
Nasal cavity and ethmoids & Nasopharynx or clivus-T4b & Pterygoid plates-T4a \\
Salivary glands & Skull base and/or pterygoid plates-T4b & Mandible-T4a \\
\hline
\end{tabular}

*Although posterior body and ramus of mandible is a content of MS

considered very advanced and T4b in the AJCC staging 7th edition. ${ }^{8}$ However, in oral cancers spread of disease above and below the mandibular notch has a bearing on resectability (Figs 7A and B) as good local control was shown by Liao et al in a subset of patients with infranotch disease extension. ${ }^{6}$ Therefore, a more practical description by the radiologist is localizing disease extension to above or below the mandibular notch. This is because infranotch MS/ITF disease is considered resectable.

In oropharyngeal cancers, medial pterygoid muscle involvement is defined as T4a while lateral pterygoid muscle, pterygoid plates, lateral nasopharynx or skull base invasion is labeled T4b. In nasopharyngeal cancers, invasion of PPS is T2b in the AJCC 7th edition and MS or ITF invasion is T4. In contrast maxillary sinus cancers with invasion of the pterygoid plates and ITF are moderately advanced or T4a. Likewise cancer of the nasal cavity or ethmoids with extension to pterygoid plates is T4a. In both maxillary and ethmoid cancers, T4b disease is nasopharyngeal or clival invasion (Table 2). ${ }^{8}$

\section{Teaching Point 3}

While abscesses tend to be contained in fascial lined spaces, such as MS, malignancy, does not respect fascial barriers and more frequently involves the larger space defined by the ITF.

\section{Pathology of the PPS}

A brief list of differential diagnoses of the prestyloid and poststyloid PPS based on the contents is given in Tables 3 and 4. To the radiologist, the PPS is a key space, as displacement of the parapharyngeal fat helps decides the epicenter of a lesion. MS masses displace the fat posteromedially while pharyngeal masses displace the fat laterally. Parotid space masses displace the fat anteromedially, whereas masses arising from the carotid sheath displace it anteriorly. Once a lesion is localized to the PPS, it has to be localized to the prestyloid or poststyloid compartment. Although the styloid is used as the landmark, a more useful pointer is the location of the carotid sheath. Poststyloid PPS masses, such as paragangliomas and schwannomas displace the carotid sheath anteriorly or anterolaterally. In contrast, the prestyloid PPS masses such pleomorphic adenomas from parapharyngeal salivary cell rests or deep lobe parotid tumors displace vessels posteriorly or posteromedially. Although this relation holds true in the vast majority, exceptions are seen in the schwannomas of

Table 3: Prestyloid PPS masses-DD (modified from Som Curtin ${ }^{1}$ )

\begin{tabular}{|c|c|c|}
\hline Content of PPS/etiology & Lesion & Lesion \\
\hline Fat & Lipoma & \\
\hline Salivary gland rests within the fat & Pleomorphic adenoma & \\
\hline Deep portion of parotid gland & Pleomorphic adenoma & $\begin{array}{l}\text { - Mucoepidermoid carcinoma } \\
\text { - Adenoid cystic carcinoma } \\
\text { - Acinic cell carcinoma }\end{array}$ \\
\hline Pterygoid venous plexus & Asymmetric plexus (pseudomass) & \\
\hline Congenital & Atypical second branchial cleft cyst & \\
\hline Spread from adjacent space & $\begin{array}{l}\text { - Pharynx (primarily tonsillitis) } \\
\text { - Parotid (acute sialadenitis) } \\
\text { - } \text { MS (odontogenic infection) } \\
\text { - Extension of abscess in lower neck }\end{array}$ & $\begin{array}{l}\text { Pharyngeal tumor: SCC, MSGC, NHL } \\
\text { Metastatic nodes: Level II, retropharyngeal } \\
\text { Parotid tumor: MEC, ACC, SCC } \\
\text { MS tumor: SCC } \\
\text { Intracranial origin: Meningioma } \\
\text { Skull base: Osteoma, chondrosarcoma, } \\
\text { chordoma }\end{array}$ \\
\hline Nerves* & $\begin{array}{l}\text { Schwannoma* } \\
\text { Neurofibroma }\end{array}$ & \\
\hline
\end{tabular}

SCC: Squamous cell carcinoma; MEC: Mucoepidermoid carcinoma; ACC: Adenoid cystic carcinoma; MSGC: Minor salivary gland carcinoma; NHL: Non-Hodgkin's lymphoma; *The rare sympathetic or IX nerve schwannoma growing anterior to carotid sheath and appearing prestyloid in location 


\begin{tabular}{lll}
\hline & Table 4: Poststyloid PPS masses: DD-based on contents (modified from Som Curtin ${ }^{1}$ ) \\
\hline Content of PPS & Lesion & Lesion \\
\hline${ }^{*}$ Nerves IX, X, XI, XII & Schwannoma (common) & Neurofibrosarcoma (rare) \\
Vessel-ICA & Neurofibroma (uncommon) & \\
& Aneurysm/pseudoaneurysm & Tumor invasion \\
& Dissection & \\
& Thrombosis & \\
& Ectasia & \\
Vessel-IJV & Medial deviation & Tumor invasion \\
& Arteriosclerotic thrombosis & \\
& Thrombophlebitis & \\
& Ectasia & \\
& Glomus jugulare & \\
& Glomus vagale & \\
& Carotid body tumor & \\
& Abscesses from adjacent spaces & From adjacent space-pharyngeal tumor: \\
& & SCC, MSGC, NHL \\
& & Metastatic nodes: Level II, retropharyngeal \\
& & Parotid tumor: MEC, SCC \\
& & Intracranial origin: Meningioma
\end{tabular}

SCC: Squamous cell carcinoma; MEC: Mucoepidermoid carcinoma; MSGC: Minor salivary gland carcinoma; NHL: Non-Hodgkin's lymphoma; *The posterior location of the X-XII nerves and paraganglionic tissue (see Table 1) is the cause for the typical anterior displacement of the carotid vessels by poststyloid masses. An exception is the rare glossopharyngeal schwannomas that can cause posterior displacement of the carotid sheath; **Sympathetic plexus though a content of the poststyloid PPS ${ }^{4}$ lies along the medial fascial wall (Table 1) and hence a schwannoma of the plexus can occasionally extend anteriorly into the prestyloid compartment displacing the carotid sheath posteriorly

the glossopharyngeal nerve (rare) or uncommonly with sympathetic plexus schwannomas which can displace the ICA posterolaterally., ${ }^{9,10}$

Differentiating masses of the prestyloid PPS requires (i) examination of the fat planes around the mass and (ii) the internal density or signal intensity characteristics. The masses arising de novo from the parapharyngeal salivary gland rests have a fat plane all around the mass (Fig. 14). These are more likely to be benign pleomorphic adenomas

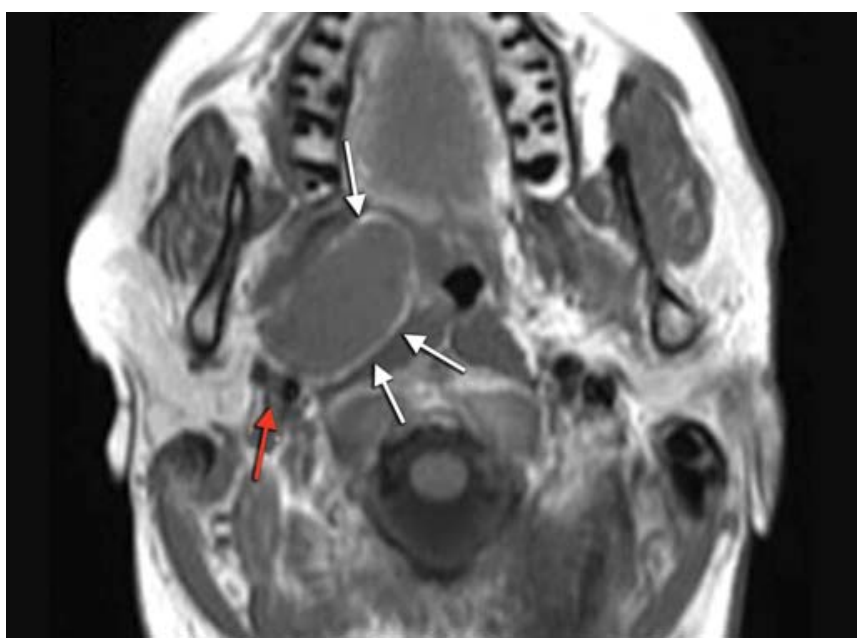

Fig. 14: T1W axial MRI section shows pleomorphic adenoma of the salivary gland cell rests in the right prestyloid PPS. Note posterior displacement of vessels (red arrow) and fat planes all around the tumor (white arrows) and malignant neoplasms are rare. Masses from the deep lobe of parotid display a fat plane only around the medial boundary of the mass, while laterally the mass merges with the parotid deep lobe and may cause widening of the stylomandibular tunnel (Fig. 15). Benign masses tend to have sharply defined margins with homogeneous solid internal architecture. On MR imaging, they are homogeneously hypointense on T1W images (Fig. 14), homogeneous and brightly hyperintense on $\mathrm{T} 2 \mathrm{~W}$ images

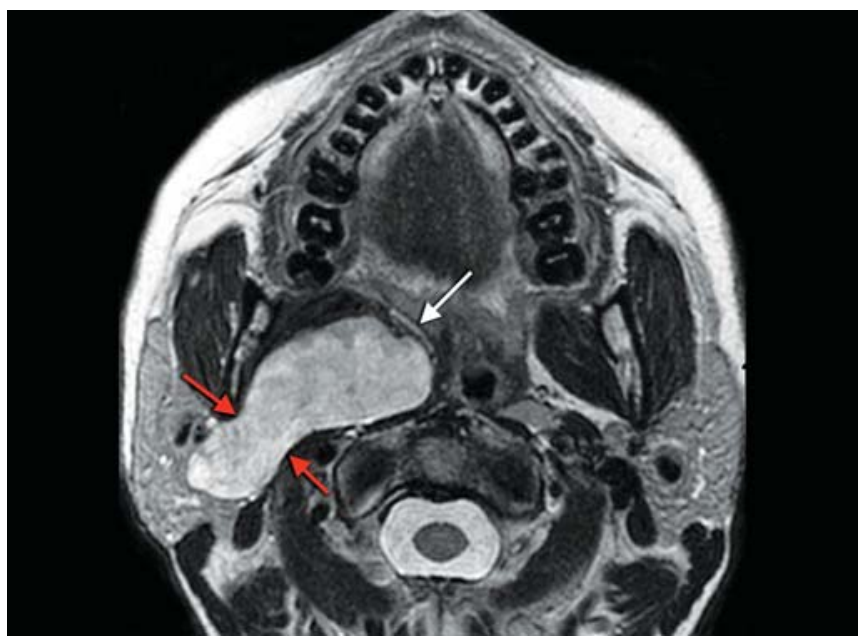

Fig. 15: T2W axial MRI shows a right deep parotid lobe tumor. Note well-defined margins, homogeneous and brightly hyperintense signal s/o benign pleomorphic adenoma. Mass is dumb-bell shaped with waist at widened stylomandibular tunnel (red arrows). No plane between tumor and deep lobe, but fat plane seen along medial margin of tumor (white arrow) 


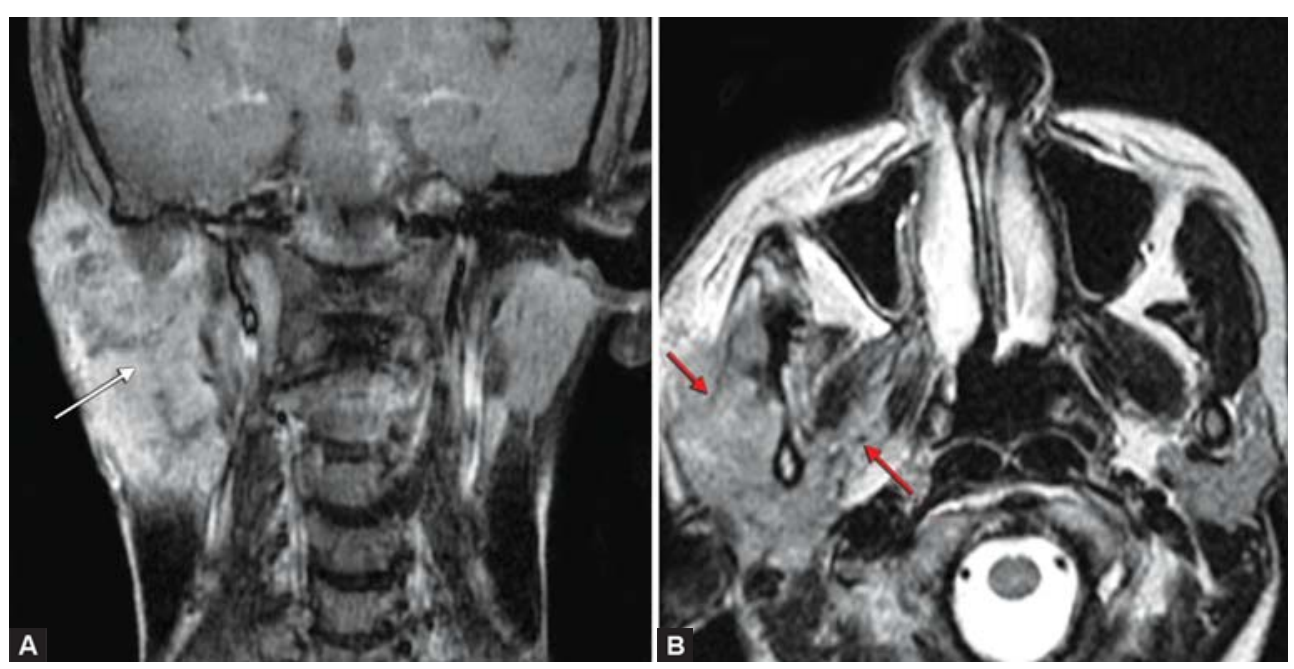

Figs 16A and B: Right parotid mucoepidermoid carcinoma. (A) Coronal T1W postcontrast MRI shows heterogeneously enhancing mass (arrow) reaching up to skin. (B) Axial T2W MRI shows intermediate signal intensity mass infiltrating pterygoid muscles (long arrow) and masseter (short arrow). Note ill-defined margins

(Fig. 15) due to high water content of serous and mucus products. They also display homogeneous enhancement. Low-grade malignant neoplasms can mimic these appearances. ${ }^{11}$

Malignant tumors have ill-defined infiltrating margins, heterogeneous signal intensity with low to intermediate signal intensity regions on $\mathrm{T} 2 \mathrm{~W}$ images (due to high cellularity) and enhance heterogeneously on contrast administration ${ }^{11}$ (Figs 16A and B). CT or MR imaging can differentiate between benign and malignant parotid masses in $90 \%$ of the cases. MRI is the optimal modality when salivary gland neoplasia is suspected, but CT is a close second choice. Another entity that needs mention is the carcinoma expleomorphic adenoma where malignant transformation occurs in a pre-existing pleomorphic adenoma or develops in a previously resected pleomorphic adenoma. This tumor has some persisting benign elements that appear bright on T2W MRI images. Malignant areas show low signal on both $\mathrm{T} 1$ and T2W sequences. ${ }^{11}$ Atypical second branchial cysts are water attenuation/intensity structures (dark on T1W and bright on T2W sequences) with thin imperceptible walls. Lipomas display fat intensity appearing bright on both $\mathrm{T} 1 \mathrm{~W}$ and $\mathrm{T} 2 \mathrm{~W}$ sequences.

The more common poststyloid PPS masses are paragangliomas and schwannomas. While both MRI and $\mathrm{CT}$ can be performed, we believe that a first line investigation with CT is advantageous over a nondynamic contrast MRI study. On dynamic postcontrast MDCT study (contrast injection with a 20-25 seconds delay) or dynamic contrast enhanced MRI, an early intensely enhancing mass with rapid wash out after 150 seconds suggests a hypervascular tumor, such as paraganglioma ${ }^{11}$ (Fig. 17). MRI is considered the modality of choice for parapharyngeal tumors due to superior soft tissue characterization and lack

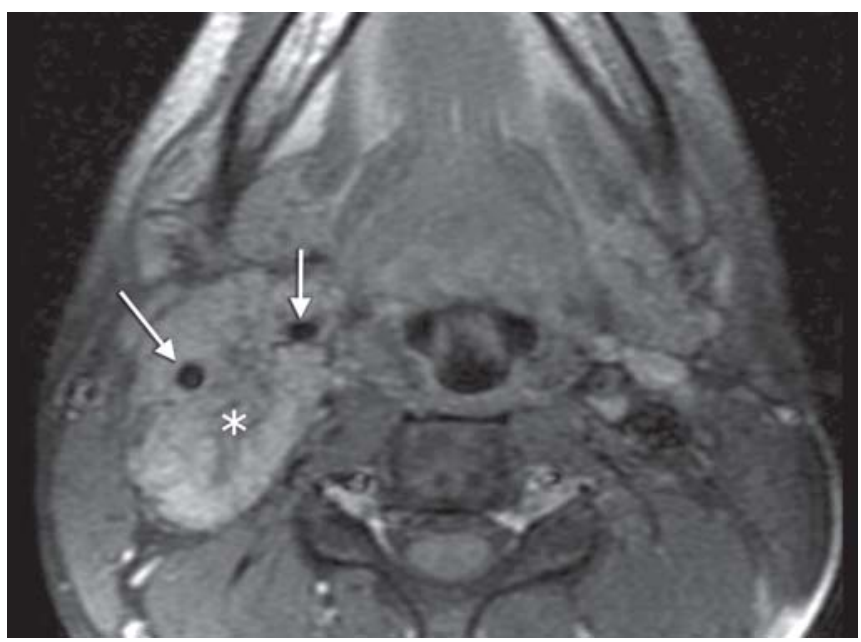

Fig. 17: Contrast-enhanced dynamic MRI shows a brightly enhancing paraganglioma encasing both ICA and ECA (arrows) indicating a Shamblin III type right-sided carotid body tumor

of ionizing radiation, ${ }^{12}$ but loses advantage when a dynamic study is not performed. MRI may display flow voids on T1W and T2W sequences seen as well-defined dark rounded or linear structures within the mass. The characteristic 'salt and pepper' appearance is infrequently seen, the salt representing bright signal intensity (areas of slow flow) on nonenhanced T1W images and the pepper indicating the flow voids seen on both T1W and T2W images.

Schwannomas can show delayed vascular enhancement by retaining contrast and hence may appear as brightly enhancing masses on contrast-enhanced nondynamic MRI study where the postcontrast images are obtained beyond 60 seconds of scanning. ${ }^{12,13}$ On the other hand, a dynamic MDCT scanning obtained within 30 seconds of scanning will not show contrast enhancement in a schwannoma (Figs 18A and B). The only exception is the rare hypervascular schwannoma. ${ }^{13}$ Large schwannomas can show necrosis and occasionally display calcification that 

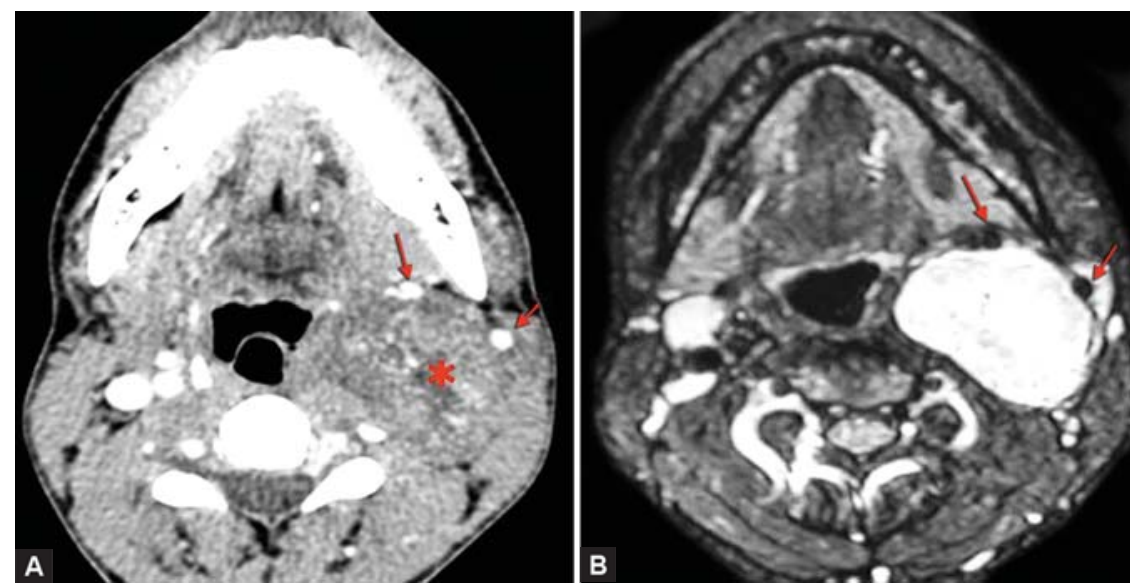

Figs 18A and B: (A) Dynamic early phase MDCT scan showing a schwannoma $\left(^{*}\right)$ without enhancement on early phase. (B) Delayed phase contrast-enhanced MRI shows intense enhancement due to contrast being retained. ICA and IJV (arrows) in A and B are displaced anteriorly (poststyloid location) and splayed (indicating origin from vagus)

may be noted as high density foci on CT and visualized as dark areas on MRI. ${ }^{1}$ Hence, while ordering MR imaging studies for PPS masses, a need for a dynamic study has to be emphasized to prevent inadvertent suboptimal imaging.

The nerve of origin of poststyloid PPS schwannomas can also be inferred from imaging features. Furukawa reported that schwannomas of the vagus nerve cause separation between the internal jugular vein and internal carotid artery (ICA) resulting in an increase in the distance between the two (Fig. 18). However, in schwannomas of the cervical sympathetic chain, no such separation was observed between the internal jugular vein and ICA. ${ }^{14}$ Others have applied the Furukawa principle and successfully diagnosed vagal schwannomas. ${ }^{13}$ Very rarely sympathetic schwannomas have been reported to splay the carotid bifurcation like carotid body tumors. Vagal schwannomas are not known to separate the ICA and ECA. ${ }^{10,13}$

Paragangliomas are classified into (i) glomus jugulare seen at the jugular foramen, (ii) carotid body tumors seen at the carotid bifurcation with typical widening of the crotch between ICA and external carotid artery and (iii) glomus vagale located at a site between skull base and carotid bifurcation. Imaging can help evaluate the circumferential contact between the ICA and the carotid body tumor (Fig. 17) helping classification into Shamblin I $\left(\leq 180^{\circ}\right)$, Shamblin II $\left(>180^{\circ}\right.$ and $\left.<270^{\circ}\right)$ and Shamblin III $\left(\geq 270^{\circ}\right) .{ }^{15}$

CT and MRI are complimentary studies in the investigation of parapharyngeal masses. A general caveat would be to perform a dynamic contrast-enhanced MDCT as first line investigation and order an MRI for prestyloid masses to differentiate between benign and malignant parotid masses. Alternately an MRI could be the initial investigation and may be the only investigation performed, if a dynamic postcontrast study is included.

\section{Teaching Point 4}

Prestyloid masses displace carotid sheath posteriorly or posteromedially and retrostyloid masses usually displace vessels anteriorly or anterolaterally (with few exceptions described above).

\section{Teaching Point 5}

When differentiating between paragangliomas and schwannomas, a dynamic postcontrast CT or MRI with delayed images is optimal as early enhancement with subsequent washout suggests paragangliomas while delayed enhancement alone suggests a schwannoma.

\section{CONCLUSION}

A clear understanding of the boundaries, contents and relationship of ITF, MS and PPS is needed for precise communication between the radiologist and clinician. Awareness of the differential diagnoses of parapharyngeal masses and their characteristic radiologic features help decide optimal imaging for accurate diagnosis.

\section{REFERENCES}

1. Som PM, Curtin HD. Parapharyngeal and masticator space lesions. In: Som PM, Curtin HD (Eds). Head neck imaging (4th ed). St Louis, Missouri, USA Elsevier Mosby 2003;1954-2002.

2. Harnsberger HR. Introduction to the Suprahyoid neck. In: Harnsberger HR (Ed). Handbook of Head \& Neck Imaging. (2nd ed), St Louis, Missouri, USA, Mosby 1995;8-28.

3. Oliver ER, Gillespie MB. Deep neck space infections. In. Paul WF, Bruce H, Valerie JL (Eds). Cummings otolaryngology head and neck surgery (4th ed). Philadelphia, PA: Elsevier Mosby 2005;1:202-03.

4. Standring S. Infratemporal and pterygopalatine fossae and temporomandibular joint. In: Strandring S (Ed). Gray’s anatomy: The anatomical basis of clinical practice (14th ed). Churchill Livingstone Elsevier 2011;527. 
5. Kim HC, Han MH, Moon MH, et al. CT and MR imaging of the buccal space: Normal anatomy and abnormalities. Korean J Radiol 2005 Jan-Mar;6(1):22-30.

6. Liao CT, Ng SH, Chang JT, et al. T4b oral cavity cancer below the mandibular notch is resectable with a favorable outcome. Oral Oncol 2007;43(6):570-79.

7. Tovi F, Hertzanu Y, Noyek AM. Infections of the infratemporal fossa: Imaging/clinical correlations. J Otolaryngol 1991 Oct;20(Suppl 3):1-11.

8. NCCN Guidelines Updates Head and Neck Cancers Version 1.2012. NCCN Clinical Practice Guidelines in Oncology (NCCN Guidelines ${ }^{\circledR}$ ). Available from: www.NCCN.org.

9. Nagamine WH, Conneely MF, Petruzzelli GJ, Hacein-Bey L. Glossopharyngeal schwannoma of the suprahyoid carotid space: Case report and discussion of the relationship to the carotid artery. Laryngoscope 2009 Apr;119(4):653-56.

10. Anil G, Tan TY. Imaging characteristics of schwannoma of the cervical sympathetic chain: A review of 12 cases. Am J Neuroradiol 2010 Sep;31(8):1408-12.

11. Som PM, Brandwein-Gensler MS. Anatomy and pathology of the salivary glands. In: Som PM, Curtin HD (Eds). Head and neck imaging (5th ed). Elsevier Mosby; 2011;2:2449-609.

12. Vogl TJ, Mack MG, Juergens M, Bergman C. Skull base tumors: Gadodiamide injection-enhanced MR imaging-drop-out effect in the early enhancement pattern of paragangliomas versus different tumors. Radiology 1993 Aug;188(2):339-46.

13. Saito DM, Glastonbury CM, El-Sayed IH, Eisele DW. Parapharyngeal space schwannomas: Preoperative imaging determination of the nerve of origin. Arch Otolaryngol Head Neck Surg 2007 Jul;133(7):662-67.

14. Furukawa M, Furukawa MK, Katoh K, Tsukuda M. Differentiation between schwannoma of the vagus nerve and schwannoma of the cervical sympathetic chain by imaging diagnosis. Laryngoscope 1996 Dec;106:1548-52.

15. Arya S, Rao V, Juvekar S, Dcruz AK. Carotid body tumors: Objective criteria to predict the Shamblin group on MR imaging. Am J Neuroradiol 2008 Aug;29(7):1349-54.

\section{ABOUT THE AUTHORS}

\section{Supreeta Arya}

Professor and Radiologist, Department of Radiodiagnosis, Tata Memorial Hospital, Mumbai, Maharashtra, India

Correspondence Address: Professor and Radiologist, Department of Radiodiagnosis, Main Building, No-127, Ground Floor, Tata Memorial Hospital, Parel, Mumbai, Maharashtra, India e-mail: supreeta.arya@gmail.com

\section{Pawan Rane}

Clinical Fellow, Department of Surgical Oncology, Head-Neck Services Tata Memorial Hospital, Mumbai, Maharashtra, India

\section{Anil D'Cruz}

Director, Professor and Surgeon, Department of Head and Neck Services Tata Memorial Hospital, Mumbai, Maharashtra, India 